\title{
Differential Electrophysiological Coupling for Positive and Negative BOLD Responses during Unilateral Hand Movements
}

\author{
Han Yuan, ${ }^{1,2}$ Christopher Perdoni, ${ }^{1}$ Lin Yang, ${ }^{1}$ and Bin $\mathrm{He}^{1}$ \\ ${ }^{1}$ Department of Biomedical Engineering, University of Minnesota, Minneapolis, Minnesota 55455 and ${ }^{2}$ Laureate Institute for Brain Research, Tulsa, \\ Oklahoma 74136
}

The coupling between neural cellular activity and blood oxygen level-dependent (BOLD) signal is of critical importance to the interpretation of fMRI. Largely unknown, however, is the degree to which different neuronal events (i.e., excitation and inhibition) maintain or disrupt the neural-hemodynamic relationship, especially in humans. In the present study, we compared local electroencephalographic (EEG) oscillations and the positive/negative BOLD responses of simultaneously recorded data from healthy human volunteers performing unilateral finger tapping at graded rates. By quantifying the single-trial modulations of EEG using source imaging, we tested for their correlation with positive BOLD response (PBR) and negative BOLD response (NBR) after coregistering their spatial locations. PBR was found to be overlapped with and correlated to the decrease of alpha $(8-13 \mathrm{~Hz})$ and beta $(13-30 \mathrm{~Hz})$ band EEG in the contralateral sensorimotor cortex. Regional EEG modulations at the sensorimotor cortex further predicted a spatially distributed and interconnected network of motor-related cortical areas. Alternatively, no significant correlation was found at the ipsilateral sensorimotor cortex between the NBR and EEG despite their spatial overlapping. This differential electrophysiological coupling of the positive and negative BOLD responses suggests that the underlying neuronal events may not only influence the direction of the signal change but also the neuralhemodynamic relationship.

\section{Introduction}

In recent years, fMRI of blood oxygen level-dependent (BOLD) responses has been widely used to investigate brain functions. Although the BOLD signal measures local changes of the hemodynamic response associated with neural processes at millimeter or submillimeter resolutions, interpretation of the BOLD response in terms of the underlying neuronal events, i.e., excitation and inhibition, is still unclear ( $\mathrm{He}$ and Liu, 2008; Logothetis, 2008).

An increasing number of studies have been conducted in an attempt to investigate the neurophysiological origin of the BOLD signal. Studies using intracranial recordings have shown that BOLD responses are most strongly related to the local changes in concurrent neuronal local field potentials (LFP) (Logothetis et al., 2001; Mukamel et al., 2005). Particularly, whereas the BOLD signal was shown to be positively correlated with LFP in highfrequency ranges $(>30 \mathrm{~Hz})$, an opposite direction of coupling

\footnotetext{
Received Oct. 9, 2010; revised May 11, 2011; accepted May 19, 2011.

Author contributions: H.Y. and B.H. designed research; H.Y., C.P., and L.Y. performed research; H.Y. and C.P. analyzed data; H.Y., C.P., and B.H. wrote the paper.

This work was supported by Grants NIH R01EB006433, NIH R01EB007920, and NSF CBET-0933067. H.Y. was supported in part by the Doctoral Dissertation Fellowship from the Graduate School of the University of Minnesota. We thank Dan Rinker for assistance in collecting the experimental data and Dr. Phil Burton for his help in experimental setup.

Correspondence should be addressed to Bin He, 7-105 NHH, 312 Church Street SE, Minneapolis, MN 55455. E-mail: binhe@umn.edu.

DOI:10.1523/JNEUROSCI.5312-10.2011

Copyright $\odot 2011$ the authors $\quad 0270-6474 / 11 / 319585-09 \$ 15.00 / 0$
}

was found with low-frequency $(5-15 \mathrm{~Hz})$ components of LFP (Mukamel et al., 2005). This finding was corroborated by other human studies using noninvasive electroencephalographic (EEG) recordings to show the negative coupling between BOLD and low-frequency EEG during a task-free state (Goldman et al., 2002; Mantini et al., 2007). Moreover, a wide range of sensory, motor, and cognitive tasks have been demonstrated to induce responses in EEG or magnetoencephalography (MEG) as local decreases of alpha $(8-13 \mathrm{~Hz}$, also known as mu rhythm) and beta (13-30 Hz) band power (Babiloni et al., 1999; Singh et al., 2002; Brookes et al., 2005). However, it is less clear how such synchronized oscillations may be related to the BOLD signal involved in human task conditions.

Toward this end, we have simultaneously recorded EEG and BOLD fMRI in a group of human subjects performing unilateral finger movements at graded rates. As previous fMRI studies have reported (Allison et al., 2000; Hayashi et al., 2008), unilateral hand movement induces positive BOLD response (PBR) in the contralateral sensorimotor cortex and negative BOLD response (NBR) in the ipsilateral area where transcallosal inhibition coincides (Purpura and Girado, 1959; Wassermann et al., 1991; Ferbert et al., 1992). Aside from an inverse correlation reported for PBR and alpha/beta band EEG (Ritter et al., 2009), the exact electrophysiological components of NBR have been controversial, ranging from a consistent correlation with decreased spiking activity (Shmuel et al., 2006) to instances where neural and hemodynamic signals may become dissociated under suppression (Devor et al., 2008; Maier et al., 2008). Still largely unknown for 
NBR is whether its relationship with electrophysiological signals is maintained in a similar fashion as PBR or whether it becomes disrupted at a macroscopic level, especially in humans.

In the present study, we hypothesized spatial coregistration as well as amplitude correlation between PBR/NBR and concurrent EEG responses. By quantifying the regional single-trial modulations of EEG using an electrophysiological source imaging approach, we found decreased EEG power in the low-frequency bands to be largely overlapped with both PBR and NBR. However, PBR showed a negative correlation with EEG in the contralateral sensorimotor cortex while no clear correlation was observed for NBR in the ipsilateral sensorimotor cortex, where the underlying neuronal events are distinct from those in the contralateral side.

\section{Materials and Methods}

Subjects. Twelve healthy volunteers participated in the study (age, $24 \pm 4$ years; seven females, five males). Half of the subjects were right-handed (handedness score, $67 \pm 8$ ) and the others were left-handed (-78 \pm 10$)$ according to the Edinburgh Handedness Inventory (Oldfield, 1971). All subjects provided written informed consent in accordance with a protocol approved by the Institutional Review Board of the University of Minnesota.

Experimental task. Subjects were instructed to tap the index finger of their left or right hand at rates of $0.5,1,1.5,2,2.5,3$, and $3.5 \mathrm{~Hz}$ in a mixed block and event-related design, as illustrated in Figure 1. The task blocks (30 s) were interleaved with rest blocks (30 s). Within a task block, subjects performed the tapping movements in periods of $2 \mathrm{~s}$ and ceased tapping during intertrial intervals of varying durations from 1 to $2 \mathrm{~s}$. The periods of tapping and rest were indicated by visual cues projected on a screen visible within the scanner. The tapping rates were consistent throughout each run and subsequently randomized and balanced across runs and subjects. To pace the tapping rates, a metronome was constantly active during each run and was delivered to subjects through headphones (Avotec).

Simultaneous EEG/fMRI recording. Continuous EEG signals were recorded using MRI-compatible BrainAmp MR Plus amplifiers (Brain Products). Sixty-two sintered $\mathrm{Ag} / \mathrm{AgCl}$ ring electrodes were mounted in a scalp cap according to the extended 10-20 international system. All electrodes were referenced to the FCz position, while a ground electrode was located below $\mathrm{Oz}$ at the midline. Two additional electrodes were placed above the left eye and on the subjects' back to monitor the electrooculagraphic and electrocardiographic signals, respectively. The impedance of all electrodes was maintained $<10 \mathrm{~K} \Omega$ throughout the recording. The signals were recorded at a sampling frequency of $5000 \mathrm{~Hz}$ with an analog filter (from 0.016 to $250 \mathrm{~Hz}$ ) at a resolution of $0.5 \mu \mathrm{V}$ (dynamic range, $16.38 \mathrm{mV}$ ). The physical landmarks (nasion and left/right preauricular points) and electrode positions were digitized using a Fastrak digitizer (Polhemus) and 3DSpace software within the SCAN software package (NeuroScan).

Imaging was performed on a Siemens TIM Trio 3T MR scanner (Siemens). Each individual anatomical MRI dataset consisted of 176 contiguous sagittal slices with $1 \mathrm{~mm}$ slice thickness (matrix size, $256 \times 256$; FOV, $256 \times 256 \mathrm{~mm}$; TR, $20 \mathrm{~ms}$; TE, $3.3 \mathrm{~ms}$ ). Whole-brain functional images with BOLD contrasts were acquired using the gradient echo planar imaging sequence (32 axial 3-mm-thick interleaved slices with 0.3 mm gap; TR, $2000 \mathrm{~ms}$; TE, $30 \mathrm{~ms}$; flip angle, 90² matrix size, $64 \times 64$; FOV, $192 \times 192 \mathrm{~mm}$ ). Each functional run began with a rest block and contained 135 volumes. Seven runs were collected for each subject and each rate condition was conducted once.

Subjects' tapping was recorded using a pressure sensor, which was part of the physiological monitoring system (ADInstruments) equipped with the scanner. The tapping signal was digitized and recorded at $1000 \mathrm{~Hz}$. The tapping rates were monitored by researchers during the experiments and also calculated during postanalysis.

EEG data analysis. The simultaneous EEG data were corrected for artifacts related to the gradient switching and cardiac ballistic effect using the template subtraction method (Allen et al., 1998, 2000) implemented in BrainVision Analyzer (Brain Products). Residual ballistocardiac artifacts in the EEG signals were removed using the independent component analysis method. The denoised data were subsequently bandpass filtered from 1 to $30 \mathrm{~Hz}$, downsampled to $250 \mathrm{~Hz}$, and rereferenced to the common average reference. The continuous EEG signals were then segmented into epochs with respect to the onsets of task cues, from -1 to $2.5 \mathrm{~s}$.

The task-induced modulations in EEG signals were quantified as power changes relative to the baseline. To examine the task effect at the sensor level, time-frequency representation of the denoised data was computed using wavelet transformation from 1 to $30 \mathrm{~Hz}$ in frequency bins of $1 \mathrm{~Hz}$ (Tallon-Baudry and Bertrand, 1999; Qin et al., 2004). The EEG was analyzed in several frequency bands (delta: $<4 \mathrm{~Hz}$; theta: $4 \mathrm{~Hz}$ to $<8 \mathrm{~Hz}$; alpha: $8-13 \mathrm{~Hz}$; and beta: $>13-30 \mathrm{~Hz}$ ). The EEG power of each frequency band was extracted, averaged, and normalized to the baseline power $(-0.5-0 \mathrm{~s})$ in terms of percentage. The resulting time course characterized the evolution of movement-related modulation in the EEG power.

EEG source estimation. To examine the locations of the task-induced modulation, we estimated the sources of EEG oscillations in the cortical space (Yuan et al., 2008, 2010). In contrast to traditional evoked potential analysis of EEG signals, the cortical activity was imaged from single-trial scalp EEGs without prior averaging. The single-trial estimation was used to capture the trial-by-trial variations and also facilitate the characterization of induced oscillation in both phase-locked and nonphase-locked responses. The current density at each elementary cortical source was estimated using the minimum norm estimate (Hämäläinen and Ilmoniemi, 1994). The locations of dipole sources were modeled on the cortical surface and their orientations were set perpendicular to the surface (Dale and Sereno, 1993; Babiloni et al., 2005; Kamousi et al., 2007; He and Liu, 2008). Individual geometric surfaces of the brain were constructed from the MRI dataset using BrainVoyager QX (Brain Innovation). The transfer matrix linking the electrode sensors and cortical sources was built using the finite element method implemented in BESA (MEGIS Software). The source power was obtained by summarizing the squared norm of the minimum norm estimates corresponding to the real and imaginary parts of the Fourier transformed time series. Percentage of power change at the source level was calculated after estimating the power during the task and baseline periods separately.

For group analysis, all dipoles from each individual were projected onto a reference brain by aligning each subject's sulcal-gyral patterns using BrainVoyager QX (Fischl et al., 1999). A fixed-effect model concatenating all subjects' responses was then implemented to assess the task-related effect on a group level. The source maps of power changes 


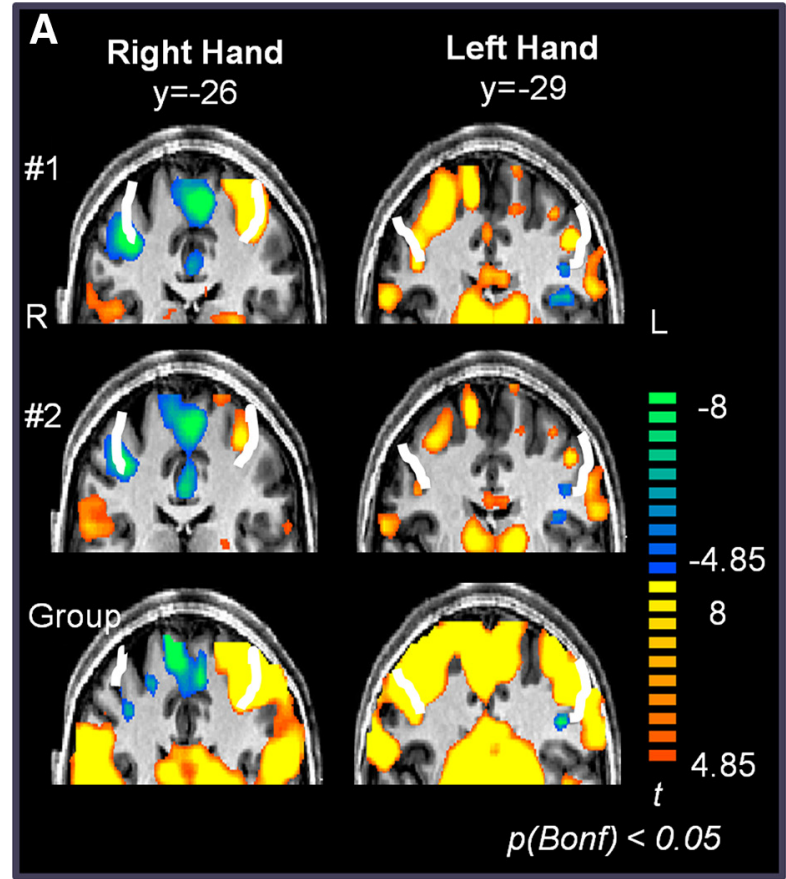

B EEG alpha source, right hand
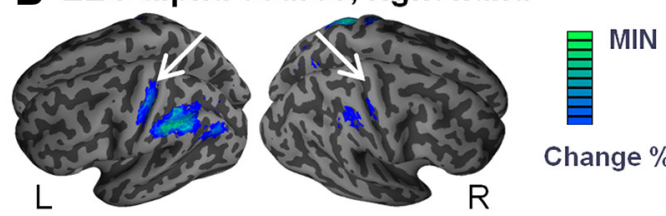

C EEG alpha source, left hand
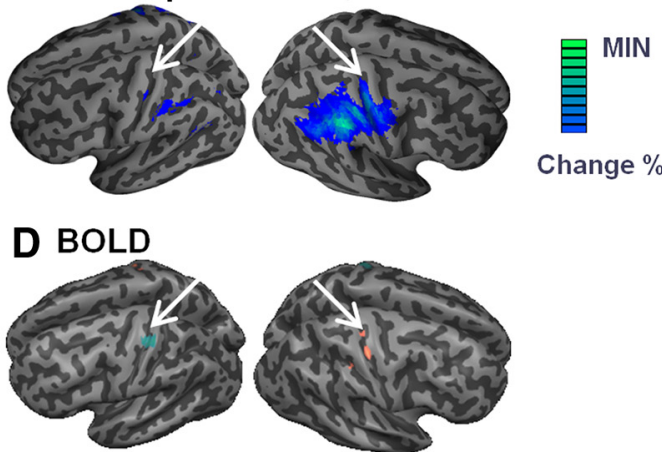

right-hand NBR and left-hand PBR left-hand NBR and right-hand PBR

Figure 2. Task-induced responses of BOLD and alpha band EEG in individuals and the group of right-handed subjects. $A$, Fixed-effect group analysis results for left- and right-hand movements. Rows 1 and 2 are from two typical subjects; the third row is the group average. White curves on the images indicate the central sulcus. Note that the contralateral PBR and ipsilateral NBR are located in close vicinity to the central sulcus. All displayed images were statistically thresholded by $p<0.05$ [after Bonferroni correction (Bonf) for whole-brain]. B, C, Fixed-effect group images of EEG power changes in the alpha band for right- and left-hand movements, respectively $(p<0.05$, Bonferroni corrected for whole-brain). White arrows indicate where the central sulcus is located on the inflated cortical surface. $\boldsymbol{D}$, Areas of conjunct PBR for contralateral movement and NBR for ipsilateral movement ( $p<0.001$, uncorrected). Only the primary sensorimotor areas were identified in the conjunction analysis of PBR and NBR. The pink region in the right hemisphere is associated with PBR during left-hand movements and NBR during right-hand movements. The light blue region in the left hemisphere is associated with PBR during right-hand movements and NBR during left-hand movements.

were thresholded at $p<0.05$ (Bonferroni corrected for whole-brain). The regions of interest (ROIs) were defined as the intersection of the group-activated areas and anatomical masks of the primary sensorimotor cortex according to the Talairach atlas database (Lancaster et al., 2000). EEG power changes from a constrained ROI were averaged within each run and one-way repeated-measures ANOVA was conducted to evaluate the rate-dependent effect.

fMRI data analysis. The fMRI data preprocessing and statistical analysis was accomplished using BrainVoyager QX (Brain Innovation). EPI images were coregistered with $\mathrm{T} 1$ anatomy and subsequently warped into the Talairach space. The preprocessing of functional data included motion correction, slice time correction, spatial smoothing using a Gaussian kernel of FWHM $=6 \mathrm{~mm}$, and temporal high-pass filtering.

The task-related effect in the BOLD signal was evaluated using a general linear model (GLM) (Friston et al., 1995). Blocks of finger movements were modeled using a boxcar function convolved with a double-gamma hemodynamic response function (Friston et al., 1998). Independent regressors were set for left- and right-hand movements. The resulting set of voxel values for each contrast constituted the map of beta values of each regressor (task vs baseline), representing the magnitudes of the BOLD responses.

For group GLM analysis, images from multiple subjects were processed using a fixed-effect model to form a group-level statistical map, which was later thresholded at $p<0.05$ (Bonferroni corrected for wholebrain). Similar to EEG, fMRI ROIs were defined based on group maps and anatomical masks. At regionally constrained ROIs, the beta values were individually estimated for each run and one-way repeatedmeasures ANOVA was conducted to evaluate the rate-dependent effect. To examine the temporal profile of the task effect, the BOLD percentage change from each ROI was calculated in reference to the average value of the four time points of the signal just before starting the task epoch and averaged across subjects for each time point.

Joint EEG-fMRI analysis. The finger tapping at various rates induced parametric changes in the EEG power as well as in the hemodynamic signals. We first examined the trial-by-trial correlation between the EEG and BOLD responses in the sensorimotor ROI. The single-trial EEG power changes were extracted and averaged across voxels within the ROI. The BOLD percentage change for each task epoch was calculated from the ROI time course and the amplitude of the BOLD response was computed by averaging the response over $12 \mathrm{~s}$ at the steady period of the change. For each subject, trials across all rate conditions were collapsed and the mean values of EEG and BOLD responses were removed to examine the cross-trial as well as the cross-rate variations. The variance from each individual's data was normalized before all subjects' data were pooled together. The linear correlation between the single-trial EEG and BOLD responses were then computed. A linear fitting was also performed between the two sets of quantities.

To further examine the temporal correlation between the EEG and BOLD responses, we intended to predict the BOLD signal using the EEG. Due to the relatively slow response time of fMRI (see Fig. 3), rather than directly correlating the BOLD signal with the time course of EEG, we convolved the EEG responses with a canonical hemodynamic response function to form a regressor in the GLM analysis. EEG power changes across all trials and all rate conditions were collapsed into one regressor and their mean values were removed to reveal the correlation based on cross-trial and cross-condition variation, as opposed to the average task effect. The EEG-informed regressor was compared with the BOLD signal at the ROI and their correlation was quantified in terms of a $t$ statistic.

To address the question of the global relationship between EEG and BOLD, we used band-specific and regionally constrained EEG regressors to map correlations with local fMRI signal across the whole-brain volume. By using GLM analysis, the EEG regressor constructed from ROI responses (described above) was compared with the BOLD signal on a voxel-by-voxel basis to show which voxels best covaried with EEG responses. In this way, it does not require any a priori information regarding fMRI activation, but instead directly identifies the areas in which BOLD signals are predicted by the EEG information originating from a particular ROI. The maps were averaged using a separate fixed-effect 
Table 1. Peak EEG response, BOLD response, and EEG-BOLD correlation in the group of right-handed subjects, with corresponding Talairach coordinates and the Euclidean distances between the peak locations

\begin{tabular}{|c|c|c|c|c|c|c|c|c|c|}
\hline & \multicolumn{2}{|l|}{ EEG } & \multicolumn{2}{|l|}{ BOLD } & \multicolumn{2}{|c|}{ EEG-BOLD correlation } & \multicolumn{3}{|c|}{ Euclidean distance (mm) } \\
\hline & Percentage change & Coordinates $(x, y, z)$ & $t$ & Coordinates $(x, y, z)$ & $t$ & Coordinates $(x, y, z)$ & EEG vs BOLD & EEG vs correlation & BOLD vs correlation \\
\hline Right hand, contralateral & -7.0 & $-37,-24,47$ & 11.0 & $-33,-29,54$ & -6.1 & $-43,-22,58$ & 9.5 & 12.7 & 12.8 \\
\hline Right hand, ipsilateral & -6.3 & $42,-22,46$ & -6.5 & $32,-26,50$ & - & - & 11.5 & - & - \\
\hline Left hand, contralateral & -8.1 & $40,-22,8$ & 9.6 & $32,-26,50$ & -7.8 & $38,-17,45$ & 9.2 & 6.16 & 11.9 \\
\hline Left hand, ipsilateral & -6.0 & $-36,-25,46$ & -5.2 & $-33,-29,54$ & - & - & 9.4 & - & - \\
\hline
\end{tabular}

$p<0.05$ (corrected) if $|t|>4.85$ or percentage change is $<-3.9 \%$.

model for the right-handed and left-handed group of subjects. The statistical images were thresholded at $p<0.001$ (uncorrected) and projected to the aligned cortical surface for display.

\section{Results}

Regional BOLD and EEG responses

In the present study, the hemodynamic and electrophysiological responses to unilateral hand movements were simultaneously recorded along with changes to the rates of movements. We first examined the spatial relationship between the EEG and BOLD responses. Figure 2, $B$ and $C$ show the group-averaged maps of EEG power changes in the alpha frequency band $(8-13 \mathrm{~Hz})$ after thresholding at the level of $p<0.05$ (Bonferroni corrected for whole-brain). As expected, the largest decrease of alpha power was observed at the contralateral sensorimotor cortex during right- or left-hand movement. At the ipsilateral hemisphere, the alpha decrease was also found to be significant, but of a smaller magnitude than the contralateral decrease. Such decreases of alpha power at the sensorimotor cortex areas were similar in both the group of right- (Fig. $2 B, C$ ) and left-handed (supplemental Fig. $2 A, B$, available at www.jneurosci.org as supplemental material) subjects.

Group maps of GLM analysis displayed PBR at the contralateral sensorimotor areas (Fig. 2A) where the alpha band EEG power showed a decrease. The inflated images of BOLD responses (see Fig. 4C) demonstrated a spatial overlapping between the decrease of alpha power and the increase of BOLD signal at the sensorimotor cortex. This spatial correspondence of PBR and alpha decrease at the contralateral sensorimotor area was found in the group of left-handed subjects as well (supplemental Fig. 4, available at www.jneurosci.org as supplemental material).

Interestingly, the primary sensorimotor areas that demonstrated positive BOLD responses to the contralateral hand movements also displayed NBR when they were ipsilateral to the performing hand. This was observed at significant levels within individual subject and group-averaged maps (Fig. $2 A$ ). Figure $2 D$ is a conjunction image of the PBR and NBR to the right- and left-hand movements $(p<0.001$, uncorrected), showing the sensorimotor cortex to be of both contralateral PBR and ipsilateral NBR (defined as the sensorimotor ROIs for BOLD). Additionally, a decrease of alpha power was found
Contralateral

B
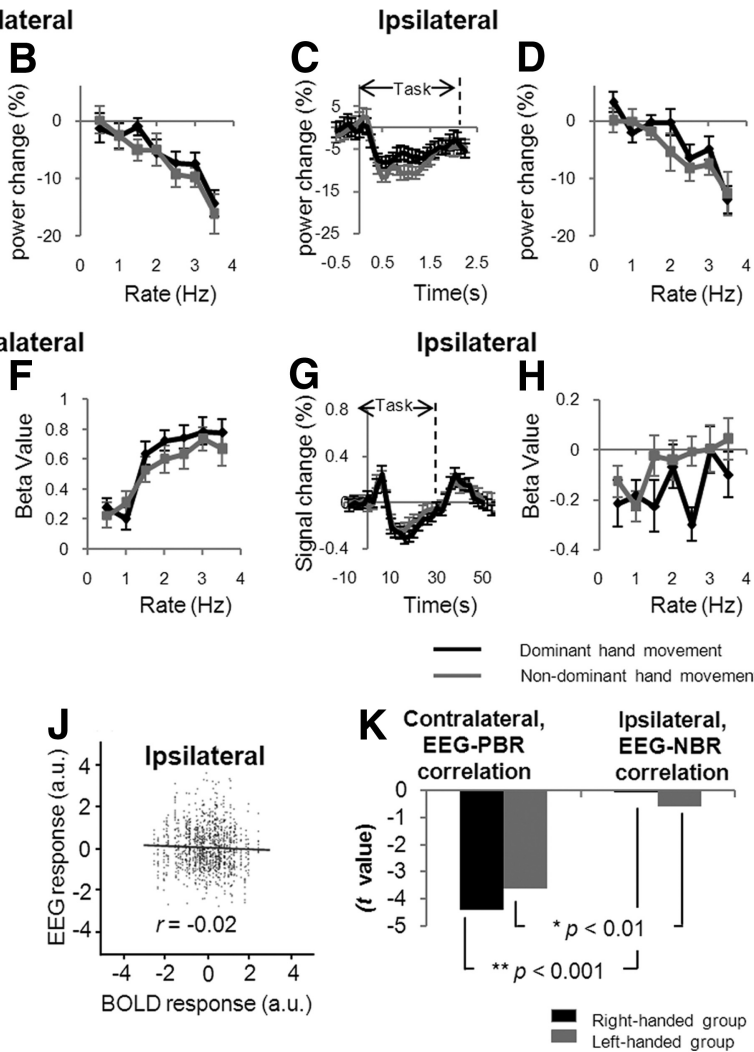

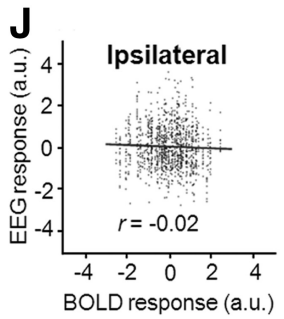

Figure 3. $\mathrm{EEG}$ response, $\mathrm{BOLD}$ response, and $\mathrm{EEG}-\mathrm{BOLD}$ correlation. $A, C, E, G$, Time courses (mean $\pm S E M$ ) of the change of $\mathrm{EE} G$ power in the alpha band $(A, C)$ and the percentage change of $\operatorname{BOLD}(\boldsymbol{E}, \boldsymbol{G})$ at the contralateral $(\boldsymbol{A}, \boldsymbol{E})$ and ipsilateral region $(\boldsymbol{C}, \boldsymbol{G})$ in response to the dominant (black) and nondominant (gray) hand movements. Note the different time scales for the BOLD and EEG responses. The BOLD lasted for $>30$ s while the EEG returned to baseline after $\sim 2 \mathrm{~S} . \boldsymbol{B}, \boldsymbol{D}, \boldsymbol{F}, \boldsymbol{H}$, Amplitudes (mean $\pm \mathrm{SEM}$ ) of $\mathrm{EEG}$ power change $(\boldsymbol{B}, \boldsymbol{D})$ and BOLD signal change $(\boldsymbol{F}, \boldsymbol{H})$ as a function of the tapping rate for the dominant (black) and nondominant (gray) hand at the contralateral $(\boldsymbol{B}, \boldsymbol{F})$ and ipsilateral $(\boldsymbol{D}, \boldsymbol{H})$ region. $\boldsymbol{I}, \boldsymbol{J}$, Scatter plots of the amplitude of the single-trial EEG responses as a function of the PBR at the contralateral $(\boldsymbol{I})$ and the NBR at the ipsilateral $(J)$ region. The plot depicts the variations in the BOLD response and EEG power decrease in single trials relative ir mean responses across all rate conditions (the mean responses were removed and variance was normalized across subjects; see linear fitting, which considered measurement errors in both coordinates. $\boldsymbol{K}$, Temporal correlation between EEG and PBR at the contralateral region compared with the EEG-NBR correlation at the ipsilateral side in both the right- (black) and left- (gray) handed groups.

at the sensorimotor cortex ipsilateral to the performing hand, overlapping with the concurrent negative BOLD responses (Fig. $2 B-D$ ). Table 1 compares the locations for the peak EEG and BOLD responses corresponding to the identified ROIs. Listed coordinates demonstrate the close vicinity between the peak responses of the two modality signals at both hemispheres (with an average Euclidean distance of $9.9 \mathrm{~mm}$ ).

\section{Parametric responses to movement rates}

To examine the rate effect of hand movements, we plotted out the task-induced responses in BOLD and EEG for each rate condition from the defined sensorimotor ROIs (Fig. 3). Data from the 
A EEG power change $\%$

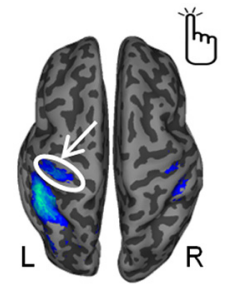

$\%$ ШШШШ MIN
B BOLD correlation with contralateral EEG in alpha band

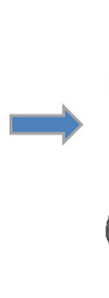

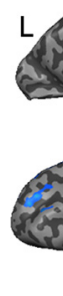
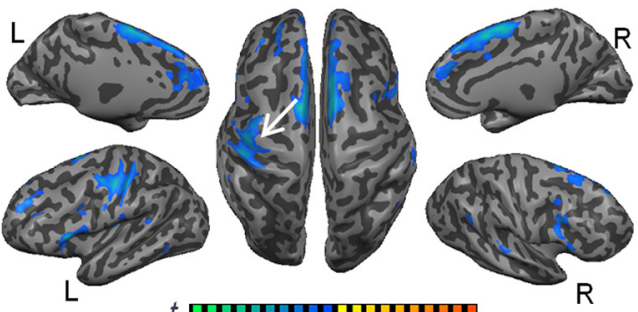

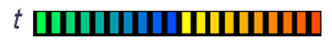

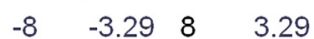

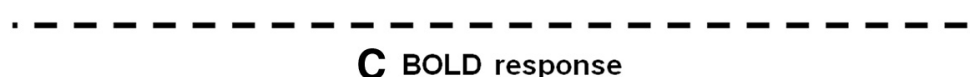

C BOLD response

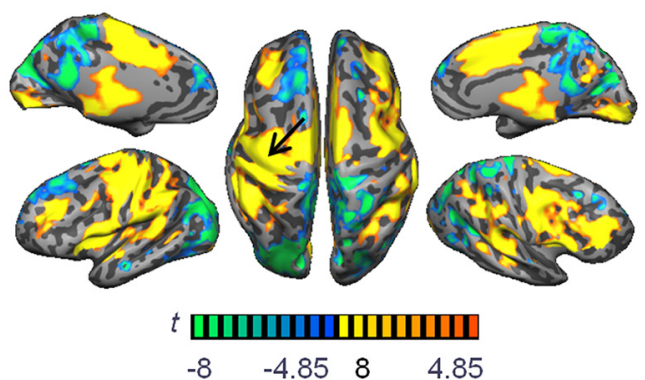

Figure 4. Correlation map between the BOLD signal and the contralateral regional EEG response during right-hand movements. The EEG regressor was derived from responses constrained within the contralateral sensorimotor ROI as indicated by the white arrow and circle $(\boldsymbol{A})$. The correlation map $(\boldsymbol{B})$ was computed by comparing the EEG regressor with the BOLD signal on a voxel-byvoxel basis and is compared with the task-effect fMRI map (C). Results were computed in the group of right-handed subjects. $A, C$, Same as in Figure 2 but displayed on an inflated cortical surface. The correlation map was thresholded at $p<0.001$ (uncorrected). The maps of EEG and BOLD responses were thresholded at $p<0.05$ (Bonferroni corrected for whole-brain). The white arrow in $\boldsymbol{B}$ and black arrow in C indicate the ROI where the EEG response was derived.

right- and left-handed groups of subjects were pooled together with regard to the movement of their dominant or nondominant hand. As shown in Figure 3B, the source power changes in the alpha band monotonically decrease as the movement rate increases for the ROIs contralateral to the performing hand. The largest amplitude of alpha power decrease was found during the fastest movement $(3.5 \mathrm{~Hz})$. This decreasing trend was prominent for both the movement of the dominant and nondominant hand. One-way repeated-measures ANOVA revealed significant rate effects for the contralateral EEG changes associated with the dominant and nondominant hand $(p=0.002$ and 0.001 , respectively). To similarly depict the trend of BOLD responses, the beta values of GLM analysis (proportional to percentage changes) were extracted and averaged from the activated sensorimotor cortex. Contrary to the EEG power changes, the amplitudes of the positive BOLD responses to the contralateral movements increased along the rate of $0.5-3.5 \mathrm{~Hz}$ (Fig. $3 F$ ). The effect of rate on the contralateral PBR was also significant for the dominant and nondominant hand ( $p=0.008$ and 0.001 , respectively). At the sensorimotor areas contralateral to the hand movement, the magnitudes of responses in BOLD and EEG power showed a common increasing trend along the movement rates, although their directions of responses were opposite (BOLD increase vs EEG power decrease).

Furthermore, in the ipsilateral sensorimotor cortex, the changes of EEG power showed a monotonic decrease with increasing rates similar to what was seen at the contralateral side (Fig. 3D). The rate effect was found to be significant for both the dominant and nondominant hand $(p=0.005$ and 0.0003 , respec- tively). However, for the negative BOLD responses at the ipsilateral area (Fig. $3 H$ ), the trend of beta values against the rates was not monotonic as was the case for the contralateral BOLD. No significant rate effect was found in the ipsilateral BOLD responses for either the dominant or nondominant hand ( $p=0.55$ and 0.24 , respectively). Interestingly, such negative BOLD responses were particularly prominent at the hemisphere ipsilateral to the dominant hand movement, where no clear trend was found between NBR and rate.

\section{EEG-BOLD correlations}

A key question in the present context is whether the task-induced responses in hemodynamic and EEG signals covary with each other. If they do, we hypothesize that such a correlated relationship should be found in brain regions where these changes are spatially overlapped. To test this hypothesis, we first compared the EEG and PBR on a trial-by-trial basis and observed a negative correlation at the contralateral sensorimotor cortex $(r=-0.19$, $\left.p<1 \times 10^{-6}\right)$ (Fig. 3I). In terms of the temporal relationship, a negative correlation was also found between the continuous BOLD signal and EEG-predicted regressor at the contralateral region in both the right- and left-handed groups (Fig. 3K). Alternatively, no significant trial-by-trial correlation was observed between the EEG and NBR at the sensorimotor ROI ipsilateral to the dominant hand movement $(r=-0.02, p>0.05)$, despite their spatial overlapping at the region. The temporal correlation between EEG and NBR was also low (Fig. $3 K$ ) and significantly different compared with the EEG-PBR correlation $(p<0.001$ and $<0.01$ for the right- and left-handed groups, respectively).

To examine the global relationship between EEG and BOLD, we used band-specific EEG regressors to map correlations with local fMRI signals across the whole-brain volume. We constrained the EEG from a defined ROI and tested where in the brain EEG responses in a certain frequency band correlated with hemodynamic responses. Results of the whole-brain correlation with alpha band EEG from the contralateral sensorimotor cortex are shown in Figure $4 B$. The EEG-BOLD-correlated regions included the same area where the EEG modulations were extracted-the contralateral sensorimotor cortex (Fig. 4A)-which indicates a coregistered spatial and temporal coupling between BOLD and EEG responses. The locations of the peak correlations are also listed in Table 1 and compared with those of the peak EEG and BOLD responses. The areas correlated to the regional EEG signal of the contralateral sensorimotor cortex also included other secondary movement-associated cortices, such as the supplemental motor area (SMA) and the bilateral premotor areas. The negative direction of the observed EEG-BOLD relationship confirmed that task-related EEG decreases of greater absolute amplitudes were associated with larger increases of BOLD signals. It is worthwhile to note that such regional EEG-informed maps directly identified the interconnected brain network associated 


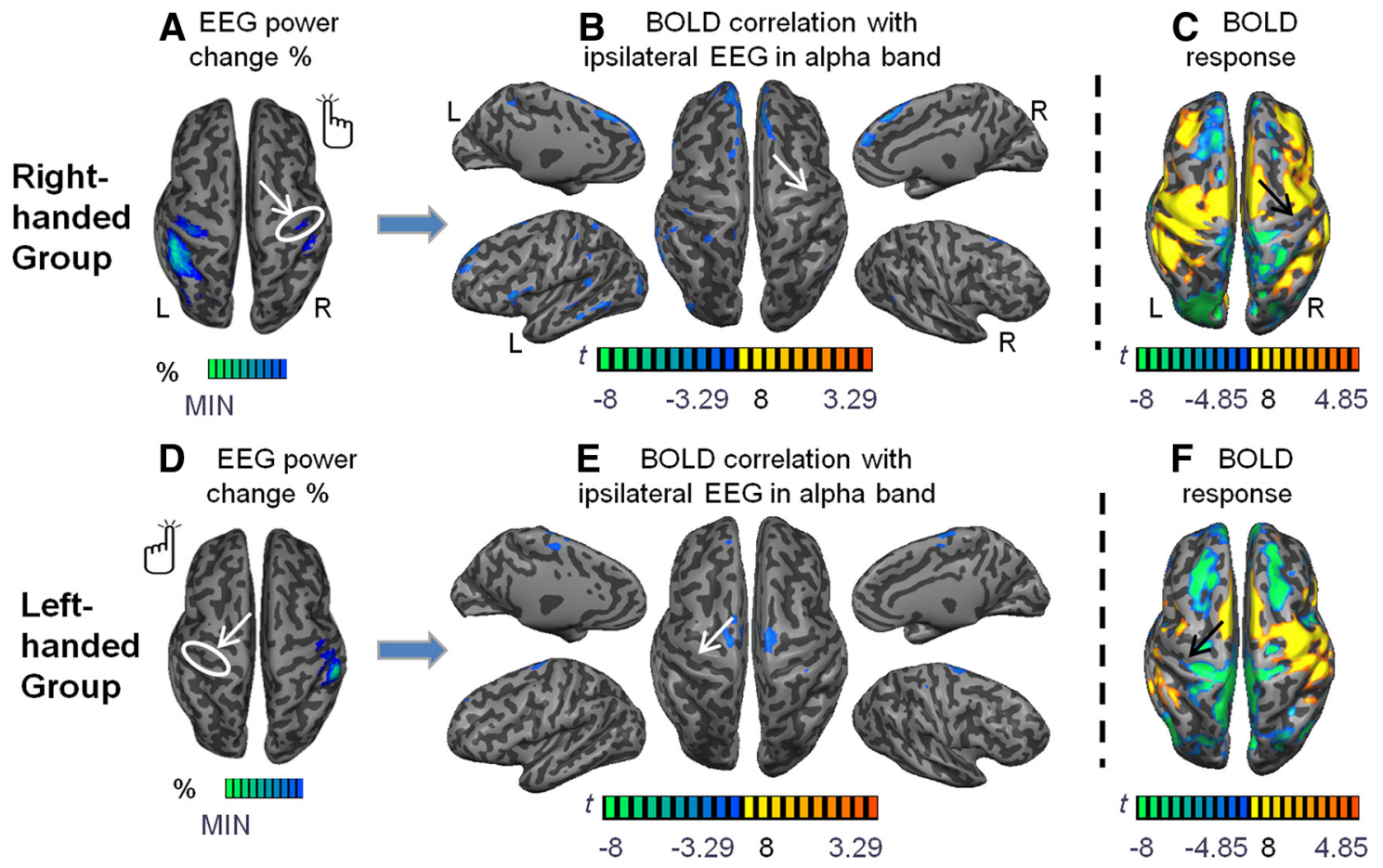

Figure 5. Correlation maps between the BOLD signal and the ipsilateral regional EEG changes during movement of the dominant hand. $\boldsymbol{B}, \boldsymbol{E}$, EEG-BOLD correlation maps associated with right-hand movements in the group of right-handed subjects $(\boldsymbol{B})$ and left-hand movements in the left-handed subjects $(\boldsymbol{E}) . \boldsymbol{A}, \boldsymbol{D}$, EEG power changes during movement of the dominant hand in the right- $(\boldsymbol{A})$ and left- $(\boldsymbol{D})$ handed groups. The EEG regressor was derived from responses constrained within the ipsilateral sensorimotor ROl, as indicated by the white arrow and circle. The correlation maps $\boldsymbol{B}$ and $\boldsymbol{E}$ are compared with the task-effect fMRI maps $\boldsymbol{C}$ and $\boldsymbol{F}$, respectively. The white arrows in $\boldsymbol{B}$ and $\boldsymbol{E}$ and black arrows in $\boldsymbol{C}$ and $\boldsymbol{F}$ indicate the ROI where the EEG response was derived.

with motor control. Although these areas were also found to be active in the task-effect fMRI maps (Fig. 4C), they were more widespread than the EEG-informed correlation maps (Fig. 4B).

For purposes of comparing, we calculated the correlation maps with regard to the EEG from the ipsilateral sensorimotor cortex. Results are shown in Figure 5 and appear very different from the contralaterally informed maps. In the right-handed group (Fig. $5 A-C$ ), the ipsilateral EEG power modulations were not found to be correlated with the concurrent negative BOLD responses at the ipsilateral side, although they were spatially overlapped. Similarly, in the group of left-handed subjects (Fig. 5D$F)$, the sensorimotor cortex ipsilateral to the dominant hand movement showed significant negative BOLD responses $(p<$ 0.05 , Bonferroni corrected for whole-brain), whereas such NBR were not correlated with the EEG modulations from the same ipsilateral sensorimotor cortex. Interestingly, the SMA and contralateral sensorimotor cortex showed significantly correlated BOLD responses to the ipsilateral EEG modulations (Fig. 5E).

In addition to the alpha EEG activity, we examined the relation of BOLD to EEG in other frequency bands, including delta, theta, and beta bands. The correlations involving these frequency bands are plotted in Figure 6 for both the PBR and NBR. Similar to alpha band activity, the EEG response in the beta band showed significant negative correlation with PBR at the contralateral sensorimotor cortex $(p<0.001$ in both the right- and left-handed groups) (Fig. $6 \mathrm{~A}$, supplemental Fig. 5, available at www.jneurosci. org as supplemental material). Meanwhile, no such correlation was found with NBR at the ipsilateral region (Fig. 6A), indicating a differential EEG-BOLD relationship with regard to the beta band EEG. Interestingly, the theta and delta band EEG activity was positively correlated with the ipsilateral NBR (Fig. $6 B, C)$ in the right-handed group $(p<0.001)$, but not, however, in the left-handed group $(p>0.05)$.

\section{Discussion}

The present study demonstrates a differential coupling for PBR and NBR with simultaneously recorded EEG oscillations in the alpha and beta frequency bands. The PBR and NBR induced by unilateral finger movements were distributed at the contralateral and ipsilateral sensorimotor cortex, where the concurrent decreases of EEG power were also localized. In spite of the spatial overlapping between regional EEG and positive/negative BOLD responses, they demonstrated distinctive relationships. Trial-bytrial amplitude variations revealed a correlation between the local decrease of EEG power and increase of BOLD signal. The singletrial EEG modulations at the sensorimotor cortex further predicted a spatially distributed and interconnected network of motor-related cortical areas. However, despite their spatial overlapping, no clear correlation was found between the ipsilateral EEG response and the concurrent NBR, suggesting different electrophysiological coupling mechanisms for PBR and NBR.

Previous studies investigating BOLD-response correlates of noninvasive EEG signals have mainly derived the electrophysiological information at the sensor level-from the sum of a few channels (Goldman et al., 2002; Laufs et al., 2003), a weighted combination of several channels (Debener et al., 2005; Eichele et al., 2005; Ritter et al., 2009), or the sum of all channels (Mantini et al., 2007; Sadaghiani et al., 2010). These groups have focused on the temporal aspect of the EEG signal, regardless of the fact that EEG itself is also a spatiotemporal dynamic process. Due to the effects of volume conduction, a single electrode records a weighted average of electrical brain activity and thus it is difficult to directly relate EEG measurements to a defined anatomical structure that may exhibit diverse neuronal processes (excitation or inhibition). Here we have used an innovative approach to overcome this confound by using an electrophysiological source 


\section{Contralateral, EEG-PBR correlation \\ Ipsilateral, EEG-NBR correlation}
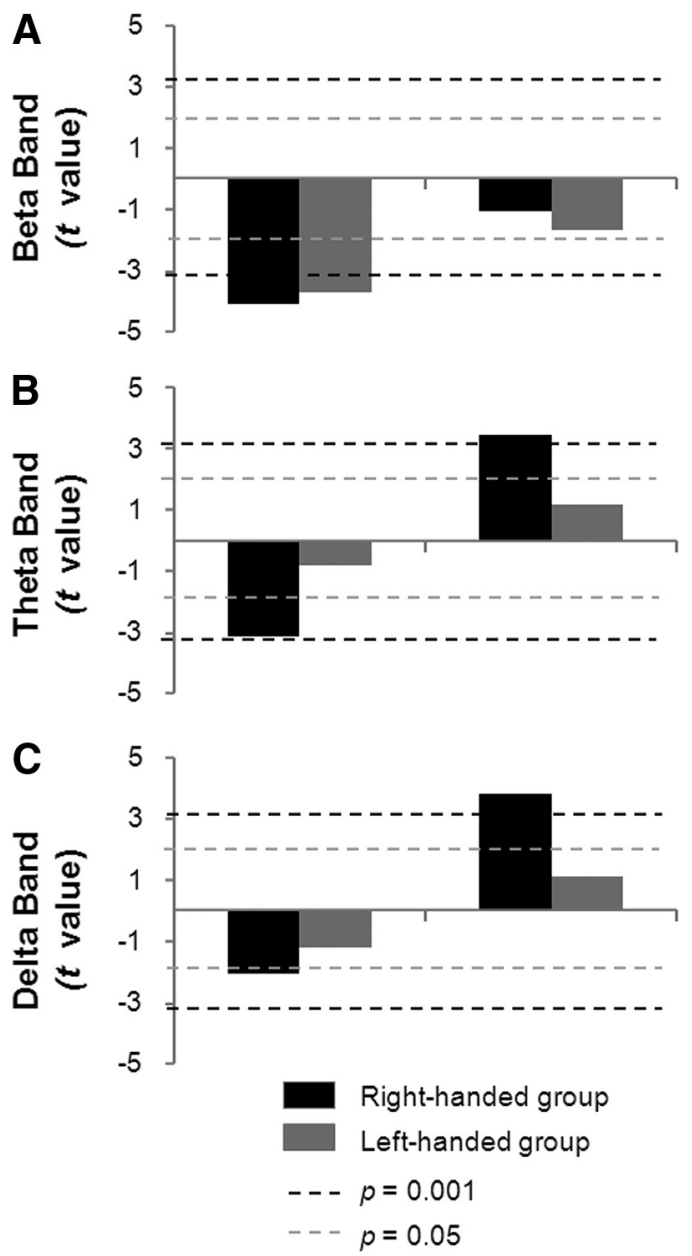

Figure 6. $\boldsymbol{A}-\boldsymbol{C}$, Correlation between BOLD and EEG in beta $(\boldsymbol{A})$, theta $(\boldsymbol{B})$, and delta $(\boldsymbol{C})$ bands. The EEG-PBR correlation at the contralateral region was compared with the EEG-NBR relationship at the ipsilateral side in both the right- (black) and left- (gray) handed groups. The black and gray dashed lines indicate the threshold of $p=0.001$ and $p=0.05$, respectively.

imaging method to spatially deconvolve the spatiotemporal dynamics of the EEG signal. We first identified the cortical regions where task-related EEG responses were found to be significant and subsequently coregistered the area of EEG modulation with that of the BOLD response. The EEG and BOLD responses were then quantified from the constrained regions and their trial-bytrial amplitude variations were compared to assess the correlation. By choosing different brain areas of interest, namely the contralateral and ipsilateral sensorimotor cortex, this regionally constrained approach allows for the examination of electrophysiological correlates for different types of BOLD responses (PBR vs NBR), which otherwise could not be delineated from globally summed EEG activity.

Our finding of the negative correlation between lowfrequency EEG oscillations and PBR at the contralateral sensorimotor cortex is in line with previous reports on the relationship between these two variables (Mukamel et al., 2005; Ritter et al., 2009; Yuan et al., 2010). Specifically, in the present study we have demonstrated their correlation under graded task-demands on a trial-by-trial basis. Such negative correlation corroborates previous findings from intracranial recordings by Mukamel et al.
(2005), though in the current case evidenced by simultaneously recorded EEG and $\mathrm{AMRI}$ data within the same group of subjects. The spatial correspondence and temporal correlation in response to tasks strongly suggests a negative functional coupling between the BOLD and low-frequency EEG oscillations. Interestingly, this is in contrast to the positive coupling between BOLD and high-frequency electrophysiological responses observed in LFPs (Logothetis et al., 2001), electrocorticogram (Lachaux et al., 2007), MEG (Brookes et al., 2005; Zumer et al., 2010), and EEG (Ball et al., 2008; Mulert et al., 2010). In addition, reciprocal changes of low- and high-frequency oscillations were found to be coupled to the increase of BOLD in V1 (Brookes et al., 2005; Hall et al., 2005; Zumer et al., 2010). These findings suggest that common neuronal events, likely neural activation, underlie the task-related hemodynamic response and multiband electrophysiological responses.

In contrast, the low-frequency EEG oscillations at the ipsilateral sensorimotor cortex demonstrated a decrease in power that was linearly related to the graded movement rates. While the contralateral power decrease was stronger than that of the ipsilateral side, bilateral regional power changes showed a monotonic decreasing trend with increasing rates. Additionally, the BOLD signal at the ipsilateral sensorimotor cortex showed a decreasing time course relative to the resting level (i.e., the negative BOLD responses). Previous transcranial magnetic stimulation studies have demonstrated transcallosal inhibition in the ipsilateral motor cortex (Wassermann et al., 1991; Ferbert et al., 1992; Meyer et al., 1995), where it appeared that a neural inhibition was spatially related to the decrease of EEG power and decrease of BOLD at the ipsilateral side. Such neural inhibition is also suggested to be a principal factor driving the negative BOLD response, rather than a pure vascular effect secondary to the neighboring positive BOLD response, as the NBR and PBR are found in the opposite sensorimotor cortices. Similar negative BOLD responses were found to be correlated with a measurable local deduction of spiking activity in V1 on a trial-by-trial basis (Shmuel et al., 2006). However, in contrast to the linear trend for EEG modulation, our study found no clear trend of NBR associated with the graded task rates, particularly at the ipsilateral side to the dominant hand movement where NBR was most prominent. Further correlational analysis based on single-trial quantities showed that the regional ipsilateral power modulation of EEG was not correlated to the NBRs from the same ipsilateral sensorimotor area. This suggests that the EEG responses and NBR at the ipsilateral sensorimotor cortex are likely a spatial coincidence rather than a functionally coupled pair.

One explanation for the differential coupling between EEG oscillations and PBR/NBR at the contralateral/ipsilateral hemisphere, respectively, is the neuronal excitation and inhibition that are unevenly related to the oscillatory and hemodynamic responses. During unilateral hand movement, the contralateral cortex is dominantly activated. However, in the ipsilateral side, there is excitation as well as transcollosal inhibition to suppress the mirror activation of the ipsilateral motor cortex for the intended hand (Nass, 1985; Allison et al., 2000). The combination of excitation and inhibition at the ipsilateral side can be varied and is dependent on the task demands (i.e., the rate). Such a difference is also supported by the asymmetric trends of ipsilateral responses with rates between the dominant and nondominant hands. Hayashi et al. (2008) hypothesized that the contralateral excitatory control during unilateral movement is approximately linearly dependent on the rates for either hand. At the ipsilateral side, the excitation is weaker than the contralateral 
side, yet still rate-dependent. However, the ipsilateral inhibitions are prominent for the dominant hand, whereas they are lower for the nondominant hand. This model in the contralateral aspect is supported by the rate-dependent effect on BOLD PBR. With regard to the ipsilateral side, this model could also explain the lack of rate-dependent effect on BOLD due to the complicated interplay of excitation and inhibition, particularly for the dominant hand. Based on this model, it is possible that the low-frequency EEG oscillations are tightly coupled to the excitatory activity, which is concurrently reflected in the positive BOLD responses. As EEG/MEG signals are mainly contributed by the large pyramidal neurons in the cerebral cortex (Hämäläinen et al., 1993), the oscillatory modulations and PBR are therefore likely to be coupled through the common underlying excitatory neuronal activities.

Although the EEG-BOLD relationship appeared to be different for the positive and negative BOLD responses, a significant functional coupling was found between the local oscillatory modulations and PBR. Based on this coupling, the regional EEG response was compared with the BOLD signal across the wholebrain volume to examine how local EEG activity is related to the widely distributed hemodynamic signal, i.e., the network-level activity. This regionally EEG-informed approach identified an intercorrelated brain network that originated from the direct functional information of the sensorimotor cortex and is directly associated with the motor task. Such an approach may act as a new imaging method to integrate the electrophysiological and hemodynamic signatures of regional event-related brain activity that may successfully identify multiple functionally relevant networks. Particularly, as shown in the present study, this multimodal analysis approach was able to identify the motor-related brain areas that were not identified by EEG alone and in a more focused and specific manner than traditional fMRI mapping would allow. This approach therefore holds promise for the tracking of much more complicated spatiotemporal dynamics of neural processes by the joint study of electrophysiological and hemodynamic imaging.

\section{References}

Allen PJ, Polizzi G, Krakow K, Fish DR, Lemieux L (1998) Identification of EEG events in the MR scanner: the problem of pulse artifact and a method for its subtraction. Neuroimage 8:229-239.

Allen PJ, Josephs O, Turner R (2000) A method for removing imaging artifact from continuous EEG recorded during functional MRI. Neuroimage 12:230-239.

Allison JD, Meador KJ, Loring DW, Figueroa RE, Wright JC (2000) Functional MRI cerebral activation and deactivation during finger movement. Neurology 54:135-142.

Babiloni C, Carducci F, Cincotti F, Rossini PM, Neuper C, Pfurtscheller G, Babiloni F (1999) Human movement-related potentials vs desynchronization of EEG alpha rhythm: a high-resolution EEG study. Neuroimage 10:658-665.

Babiloni F, Cincotti F, Babiloni C, Carducci F, Mattia D, Astolfi L, Basilisco A, Rossini PM, Ding L, Ni Y, Cheng J, Christine K, Sweeney J, He B (2005) Estimation of the cortical functional connectivity with the multimodal integration of high-resolution EEG and fMRI data by directed transfer function. Neuroimage 24:118-131.

Ball T, Demandt E, Mutschler I, Neitzel E, Mehring C, Vogt K, Aertsen A, Schulze-Bonhage A (2008) Movement related activity in the high gamma range of the human EEG. Neuroimage 41:302-310.

Brookes MJ, Gibson AM, Hall SD, Furlong PL, Barnes GR, Hillebrand A, Singh KD, Holliday IE, Francis ST, Morris PG (2005) GLMbeamformer method demonstrates stationary field, alpha ERD and gamma ERS co-localisation with fMRI BOLD response in visual cortex. Neuroimage 26:302-308.

Dale AM, Sereno MI (1993) Improved localization of cortical activity by combining EEG and MEG with MRI cortical surface reconstruction: a linear approach. J Cogn Neurosci 5:162-176.

Debener S, Ullsperger M, Siegel M, Fiehler K, von Cramon DY, Engel AK (2005) Trial-by-trial coupling of concurrent electroencephalogram and functional magnetic resonance imaging identifies the dynamics of performance monitoring. J Neurosci 25:11730-11737.

Devor A, Hillman EM, Tian P, Waeber C, Teng IC, Ruvinskaya L, Shalinsky MH, Zhu H, Haslinger RH, Narayanan SN, Ulbert I, Dunn AK, Lo EH, Rosen BR, Dale AM, Kleinfeld D, Boas DA (2008) Stimulus-induced changes in blood flow and 2-deoxyglucose uptake dissociate in ipsilateral somatosensory cortex. J Neurosci 28:14347-14357.

Eichele T, Specht K, Moosmann M, Jongsma ML, Quiroga RQ, Nordby H, Hugdahl K (2005) Assessing the spatiotemporal evolution of neuronal activation with single-trial event-related potentials and functional MRI. Proc Natl Acad Sci U S A 102:17798-17803.

Ferbert A, Priori A, Rothwell JC, Day BL, Colebatch JG, Marsden CD (1992) Interhemispheric inhibition of the human motor cortex. J Physiol 453:525-546.

Fischl B, Sereno MI, Tootell RB, Dale AM (1999) High-resolution intersubject averaging and a coordinate system for the cortical surface. Hum Brain Mapp 8:272-284.

Friston KJ, Holmes AP, Worsley KJ, Poline JB, Frith CD, Frackowiak RS (1995) Statistical parametric maps in functional imaging: a general linear approach. Hum Brain Mapp 2:189-210.

Friston KJ, Fletcher P, Josephs O, Holmes A, Rugg MD, Turner R (1998) Event-related fMRI: characterizing differential responses. Neuroimage 7:30-40.

Goldman RI, Stern JM, Engel J Jr, Cohen MS (2002) Simultaneous EEG and fMRI of the alpha rhythm. Neuroreport 13:2487-2492.

Hall SD, Holliday IE, Hillebrand A, Singh KD, Furlong PL, Hadjipapas A, Barnes GR (2005) The missing link: analogous human and primate cortical gamma oscillations. Neuroimage 26:13-17.

Hämäläinen MS, Ilmoniemi RJ (1994) Interpreting magnetic fields of the brain: minimum norm estimates. Med Biol Eng Comput 32:35-42.

Hämäläinen MS, Hari R, Ilmoniemi RJ, Knuutila J, Lounasmaa OV (1993) Magnetoencephalography: theory, instrumentation, and applications to noninvasive studies of the working human brain. Rev Mod Phys 65:413-497.

Hayashi MJ, Saito DN, Aramaki Y, Asai T, Fujibayashi Y, Sadato N (2008) Hemispheric asymmetry of frequency-dependent suppression in the ipsilateral primary motor cortex during finger movement: a functional magnetic resonance imaging study. Cereb Cortex 18:2932-2940.

He B, Liu Z (2008) Multimodal functional neuroimaging: integrating functional MRI and EEG/MEG. IEEE Rev Biomed Eng 1:23-40.

Kamousi B, Amini AN, He B (2007) Classification of motor imagery by means of cortical current density estimation and Von Neumann entropy. J Neural Eng 4:17-25.

Lachaux JP, Fonlupt P, Kahane P, Minotti L, Hoffmann D, Bertrand O, Baciu M (2007) Relationship between task-related gamma oscillations and BOLD signal: new insights from combined fMRI and intracranial EEG. Hum Brain Mapp 28:1368-1375.

Lancaster JL, Woldorff MG, Parsons LM, Liotti M, Freitas CS, Rainey L, Kochunov PV, Nickerson D, Mikiten SA, Fox PT (2000) Automated Talairach atlas labels for functional brain mapping. Hum Brain Mapp 10:120-131.

Laufs H, Krakow K, Sterzer P, Eger E, Beyerle A, Salek-Haddadi A, Kleinschmidt A (2003) Electroencephalographic signatures of attentional and cognitive default modes in spontaneous brain activity fluctuations at rest. Proc Natl Acad Sci U S A 100:11053-11058.

Logothetis NK (2008) What we can do and what we cannot do with fMRI. Nature 453:869-878.

Logothetis NK, Pauls J, Augath M, Trinath T, Oeltermann A (2001) Neurophysiological investigation of the basis of the fMRI signal. Nature 412:150-157.

Maier A, Wilke M, Aura C, Zhu C, Ye FQ, Leopold DA (2008) Divergence of fMRI and neural signals in V1 during perceptual suppression in the awake monkey. Nat Neurosci 11:1193-1200.

Mantini D, Perrucci MG, Del Gratta C, Romani GL, Corbetta M (2007) Electrophysiological signatures of resting state networks in the human brain. Proc Natl Acad Sci U S A 104:13170-13175.

Meyer BU, Röricht S, Gräfin von Einsiedel H, Kruggel F, Weindl A (1995) Inhibitory and excitatory interhemispheric transfers between motor cor- 
tical areas in normal humans and patients with abnormalities of the corpus callosum. Brain 118:429-440.

Mukamel R, Gelbard H, Arieli A, Hasson U, Fried I, Malach R (2005) Coupling between neuronal firing, field potentials, and FMRI in human auditory cortex. Science 309:951-954.

Mulert C, Leicht G, Hepp P, Kirsch V, Karch S, Pogarell O, Reiser M, Hegerl U, Jäger L, Moller HJ, McCarley RW (2010) Single-trial coupling of the gamma-band response and the corresponding BOLD signal. Neuroimage 49:2238-2247.

Nass R (1985) Mirror movement asymmetries in congenital hemiparesis: the inhibition hypothesis revisited. Neurology 35:1059-1062.

Oldfield RC (1971) The assessment and analysis of handedness: the Edinburgh inventory. Neuropsychologia 9:97-113.

Purpura DP, Girado M (1959) Synaptic mechanisms involved in transcallosal activation of cortico-spinal neurones. Arch Ital Biol 47:111-139.

Qin L, Ding L, He B (2004) Motor imagery classification by means of source analysis for brain-computer interface applications. J Neural Eng 1:135-141.

Ritter P, Moosmann M, Villringer A (2009) Rolandic alpha and beta EEG rhythms' strengths are inversely related to fMRI-BOLD signal in primary somatosensory and motor cortex. Hum Brain Mapp 30:1168-1187.

Sadaghiani S, Scheeringa R, Lehongre K, Morillon B, Giraud AL, Kleinschmidt A (2010) Intrinsic connectivity networks, alpha oscillations, and tonic alertness: a simultaneous electroencephalography/functional magnetic resonance imaging study. J Neurosci 30:10243-10250.

Shmuel A, Augath M, Oeltermann A, Logothetis NK (2006) Negative functional MRI response correlates with decreases in neuronal activity in monkey visual area V1. Nat Neurosci 9:569-577.

Singh KD, Barnes GR, Hillebrand A, Forde EM, Williams AL (2002) Taskrelated changes in cortical synchronization are spatially coincident with the hemodynamic response. Neuroimage 16:103-114.

Tallon-Baudry C, Bertrand O (1999) Oscillatory gamma activity in humans and its role in object representation. Trends Cogn Sci 3:151-162.

Wassermann EM, Fuhr P, Cohen LG, Hallett M (1991) Effects of transcranial magnetic stimulation on ipsilateral muscles. Neurology 41:1795-1799.

Yuan H, Doud A, Gururajan A, He B (2008) Cortical imaging of eventrelated (de)synchronization during online control of brain-computer interface using minimum-norm estimates in frequency domain. IEEE Trans Neural Syst Rehabil Eng 16:425-431.

Yuan H, Liu T, Szarkowski R, Rios C, Ashe J, He B (2010) Negative covariation between task-related responses in alpha/beta-band activity and BOLD in human sensorimotor cortex: an EEG and fMRI study of motor imagery and movements. Neuroimage 49:2596-2606.

Zumer JM, Brookes MJ, Stevenson CM, Francis ST, Morris PG (2010) Relating BOLD fMRI and neural oscillations through convolution and optimal linear weighting. Neuroimage 49:1479-1489. 